\title{
The Ausback Study: An Audit of Low Back Pain Outcomes in Australian Musculoskeletal Medicine Practices
}

Dr Scott Masters

Caloundra Spinal and Sports Medicine Centre, Caloundra, Australia

\author{
A/Prof Michael Yelland, \\ School of Medicine, Griffith University, Logan, Australia
}

Mr Kent Sweeting

Performance Podiatry and Physiotherapy, West End, Brisbane, Australia

Prof Philip Schluter

Auckland University of Technology, School of Public Health and Psychosocial Studies, Auckland, New Zealand; and The University of Queensland, School of Nursing and Midwifery, Queensland, Australia

Dr Geoff Harding

Sandgate Spinal Medicine Clinic, Sandgate, Brisbane, Australia

Corresponding author:

Dr Scott Masters

Caloundra Spinal and Sports Medicine Centre, 
39 Minchinton St

Caloundra, Q 4551

Australia

Ph:+61- 7-5491-1144

Fax: +61- 7-5491-1253

Email: standeven@optusnet.com.au 


\begin{abstract}
Background: The major influences on musculoskeletal medicine practitioners in Australia in the last 20 years have been from an eclectic mix of local and international experts and evidence from many disciplines. This has resulted in practitioners with a multimodal approach that combines evidence with pragmatism. The effectiveness of this combined approach on Australian patients with low back pain needs testing to see if it has broader application.
\end{abstract}

Objectives: This study aimed to measure the effectiveness of a multimodal approach practiced by musculoskeletal medicine practitioners in a private clinic setting supported by a referral network of allied health professionals.

Methods: This non-randomised non-consecutive audit comprised 75 patients with low back pain +/- leg pain from three musculoskeletal medicine practices in Queensland, Australia. Pain, disability, general health and global response were followed in participants for six months.

Results: For this cohort, the mean (SD) age was 45 (10) years and the median (IQR) duration of low back pain was $4(0.5-8)$ years. The follow-up rate at six months was 75\%. Clinically and statistically significant reductions in mean scores from baseline to six months were noted for pain from 5.5 (95\% confidence interval [CI]: 5.0, 6.0) to 2.9 (95\% CI:2.3, 3.5) at six months and for Roland-Morris disability questionnaire from 12.2 
(95\% CI:11.0, 13.3) to 7.7 (95\% CI:6.2, 9.2). At six months, 51\% were much improved or very much improved. Better reductions in pain and disability were noted in subgroups that were not working, that had pain duration up to three months and a past history of one to two episodes of low back pain.

Conclusions: The improvements in pain and disability at six months observed in this study were similar to those observed for other conservative treatment methods for chronic low back pain.

KEYWORDS: chronic low back pain, musculoskeletal, multimodal, pain, disability 


\section{INTRODUCTION}

The second most common reason for the Australian population visiting their general practitioner is musculoskeletal pain, with $50 \%$ of these people suffering from low back pain (LBP).[1] Low back pain is the most common cause of musculoskeletal disability in Australia and there have been calls for it to be targeted for research[2] and to be established as a National Health Priority Area.[3] Significantly, nearly a third of those experiencing an episode of acute LBP do not fully recover or return to work 12 months after the initial onset of pain.[4]

Musculoskeletal medicine in Australia, as in other countries, has drawn from many disciplines. Historically, the major influences have come from medical practitioners using manipulation and injection techniques taught by James Cyriax, Stoddard’s Osteopathic techniques, Murtagh and Kenna's synthesis of Maitland's (manipulative physiotherapy) techniques, French (Maigne), Swedish (Dvorak), and then North American Osteopathic techniques (Bourdillon/Greenman/Mitchell).

Beginning in 1998, the Australian National Musculoskeletal Medicine Initiative (NMMI) trial used an evidence based approach to assess treatment of acute musculoskeletal pain conditions.[5] The clinics employed a variety of manual therapy and injection techniques to treat acute conditions. Importantly, however, they also utilized Indahl's approach which relied solely on education and reassurance.[6] 
The NMMI revealed that evidenced based care of acute LBP from doctors trained in musculoskeletal medicine resulted in greater rates of full recovery and less need for continuing care compared to usual care from motivated general practitioners.[5]

For subacute LBP, there is moderate evidence that a multi-disciplinary biopsychosocial rehabilitation is moderately effective.[7] For acute and subacute LBP in Sweden, the STAYAC algorithm applied in primary care showed early evidence for the effectiveness of a multimodal, multidisciplinary program implemented in a structured algorithm,[8] but data on its effectiveness for chronic LBP have not been published. The STAYAC approach, though still pragmatic and flexible, utilizes a multimodal and multidisciplinary algorithm giving it a somewhat more formal schema to follow.

With respect to persistent LBP there is little evidence for the effectiveness of single therapies used for chronic LBP.[9] Further, for a multimodal and multidisciplinary musculoskeletal medicine approach the data is lacking, particularly in Australia. Authors preparing guidelines have lamented this lack of data.[10] As a follow-on to the NMMI, the authors designed a study in Australian musculoskeletal medicine clinics to describe the outcomes of a multimodal treatment program combining the methods of the NMMI[5] with the STAYAC algorithm[11] on patients with acute or chronic LBP with or without radicular leg pain.

\section{METHODS}




\section{Study Design}

A non-randomised non-consecutive case series audit from June 2008 to June 2009.

\section{Participants and Settings}

The study was conducted across three community-based musculoskeletal medicine clinics in south-east Queensland, Australia. These clinics were independently owned and operated and offered direct access for patients and for patients referred by other practitioners. The musculoskeletal doctors working in them had a multimodal approach to treatment supported by a network of allied health professionals. Inclusion criteria were LBP with or without pain radiating to one or both legs not requiring surgical or rheumatological care in adults aged 18 to 70 years. In selecting these we were guided by the Swedish STAYAC study criteria [8] but did not place limits on the duration of the back pain. We also increased the upper age limit as age-related differences in their conditions and responses were considered unlikely to materially affect the results. These adjustments assisted recruitment and statistical power. Acute pain was defined as pain present for up to three months and chronic pain at pain present for longer than three months. The patient had to be new to the treating doctor for the current episode of pain. Patients with red flag conditions (e.g. tumors, fractures and inflammatory arthritis) and pregnant women were excluded.

The sample size was determined by available resources rather than an a priori sample size calculation. Participants were recruited from June 2007 to June 2008 through the private practices of the three treating musculoskeletal medicine doctors. Applicant eligibility was assessed by a clinical assessment by the treating doctor. The study protocol was 
explained to eligible patients and their written consent obtained by another staff member at the practice. Ethics approval for the study was given by the Griffith University Human Research Ethics Committee in Brisbane, Australia.

\section{Patient Evaluation}

The primary outcome measures were back pain intensity and disability. Usual pain intensity in the last week was measured on a numerical rating scale[12] from zero to 100 . This has been shown to be a consistent measure of pain comparable to the visual analogue scale. The minimum clinically important difference in pain was taken as 17.5 points, based on a wide range of chronic pain studies.[13] The disability measure was the Roland-Morris low back pain and disability questionnaire (RMDQ),[1] comprised of 24 Yes/No questions on the effect of back pain on everyday activities. The criterion for treatment success was a decrease of three points in the Roland-Morris low back pain and disability questionnaire. This was the minimum clinically important change (MCIC) calculated by Riddle, Stratford \& Binkley.[14]

Secondary outcome measures included the Disability Rating Index,[8,15] SF-12 health survey,[16] and seven-point Likert scales for treatment satisfaction (from extremely dissatisfied to extremely satisfied)[17] and Patient Global Impression of Change (PGIC) (from very much worse to very much better).[18,19] All secondary outcome measures are reliable, valid and sensitive outcome measures.[8,15-17] 
The pain, disability and SF-12 measures were recorded by questionnaire administered by mail with telephone backup at inception, one, three and six months. The treatment satisfaction and PGIC measures were taken at one, three and six months. Both before and during the treatment period a record was made of all back pain related investigations, treatment and referrals.

\section{Treatment}

Therapies used with participants in this study included education/counselling, manipulation, mobilisation, positional release techniques, stretching and strengthening programs, medication, joint injections with corticosteroid and local anaesthetic, trigger point injections with local anaesthetic and prolotherapy injections. One doctor also performed caudal epidural injections for radicular leg pain.

The choice and administration of treatments was not dictated by any strict protocol or algorithm, rather they were influenced by the training and experience of the treating doctors and by patient preference. The doctors' training included Australian and New Zealand based Graduate Diplomas of Musculoskeletal Medicine and numerous short courses and conferences on manual therapies, injection techniques for muscle, joints and ligaments, exercise therapy and chronic pain management. They had all been trained in the evidence-based guidelines and protocols for LBP from the Australian NMMI[5] and in the STAYAC algorithm for musculoskeletal pain.[5,9] Most commonly treatment is multimodal often combining education, manual therapy, medication, injections and cognitive behavioural therapy (CBT)/psychotherapy. Both approaches recognize the 
limited ability of clinical assessment and investigations to make a definitive pathomorphological diagnosis. The NMMI approach commonly uses the diagnostic term somatic pain of unknown origin,[5] whereas the STAYAC algorithm uses the term 'dysfunction'.[8] Both approaches attempt to manage the patients' fears and misconceptions with confident explanation and encouragement for the patient to resume or restore normal activities through simple exercises and graded activity. The STAYAC approach has a much greater emphasis on pragmatic trials of manual therapy and injection treatments, and working closely with physical therapists and psychologists in an integrative approach.

\section{Statistical analysis}

Frequency and percents were used to describe patient characteristics, number of consultations, the treatments or actions taken and the global assessments of treatment success. For descriptive results, we reported mean (SD) and range for normal distributions and median (Q1, Q3) values for skewed distributions and we refer to (Q1, Q3) as the inter-quartile range (IQR). Spearman's correlation was used to measure the degree of association between two continuous variables. Longitudinal data analysis of the pain, disability and SF-12 scores were conducted using normal generalized estimating equation (GEE) models, with unstructured correlation matrix and robust Huber-White sandwich variance estimators. As trends over time could not be assumed to be linear, and time itself is such a vital component, all analyses included time as a classification variable. Scores at inception were the taken to be reference category from which all other 
means at other follow-up times were compared. All analyses were undertaken and figures drawn using Stata version 10.0 (StataCorp, College Station, TX, USA), and $\alpha=0.05$ defined statistical significance for all tests.

\section{RESULTS}

\section{Patient characteristics}

The characteristics of the patient cohort are summarized in table 1 . There was a predominance of females. Over $80 \%$ were working and had chronic pain. Over $70 \%$ reported morning stiffness, leg pain and previous episodes of back pain; however a diagnosis of radicular pain was made in only $28 \%$. Twenty percent of patients were smokers. The mean (SD) age of patients was 45 (10) years with a range of 20 to 67 years, and the median (IQR) duration of LBP was 4 (0.5-8) years. The median (IQR) number of consultations with the doctors in the study period was 3 (1- 12).

\section{Table 1: Characteristics of the patient cohort at inception $(n=75)$}

\begin{tabular}{ll}
\hline Characteristic & n (\%) \\
\hline Female & $43(57)$ \\
Working & $64(85)$ \\
Chronic pain & $61(81)$ \\
Previous episodes of LBP & $58(77)$ \\
Leg pain & $51(71)$ \\
Radicular pain & $21(28)$ \\
Morning stiffness & $59(79)$ \\
\hline
\end{tabular}




\section{Treatment}

The spectrum and frequency of therapies and medications used and referrals made during the study period are described in table 2 . The most commonly used treatment was education or counselling (99\%). Next in order were home stretching (60\%) and manipulation/mobilisation (55\%). The most commonly prescribed medications were non-steroidal anti-inflammatory drugs (32\%) followed by COX-2 inhibitors (24\%) and paracetamol (23\%). One-third of patients were referred to physiotherapists, all by two of the three study doctors. A total of $10 \%$ were referred to other therapists or specialists.

Table 2. Therapies and medications used and referrals made for the 75 patients during the study period, presented in descending order of frequency. Percentages total over 100 as for therapies and medications as patients could have more than one treatment or action for their condition.

\begin{tabular}{ll}
\hline Treatment/Action & \multicolumn{1}{c}{$\mathbf{n ( \% )}$} \\
\hline Therapies & $74(99)$ \\
Education/counselling & $45(60)$ \\
Home stretching & $41(55)$ \\
Manipulation/mobilisation & $26(35)$ \\
Steroid \& LA injection & $18(24)$ \\
Physical training program
\end{tabular}




\begin{tabular}{ll}
\hline LA injection & $14(19)$ \\
Prolotherapy injections & $9(12)$ \\
Stretching by doctor/therapist & $8(11)$ \\
Caudal epidural & $5(7)$ \\
Traction & $2(3)$ \\
Medications & \\
NSAID & $24(32)$ \\
COX 2 inhibitor & $18(24)$ \\
Paracetamol & $17(23)$ \\
Weak opioid & $11(15)$ \\
Strong opioid & \\
Massage therapist & $1(1)$ \\
Other & $1(1)$ \\
&
\end{tabular}

\section{Outcomes}

The mean pain score at inception was 55.2 (95\% confidence interval [CI]: 50.1, 60.3). This reduced progressively and significantly to 28.0 (95\% CI: 22.7, 33.4, $\mathrm{p}<0.001$ ) by 
three months. The mean pain score at six months was also significantly lower than inception at 28.7 (95\% CI: 22.9, 34.5. p<0.001) (Fig. 1).

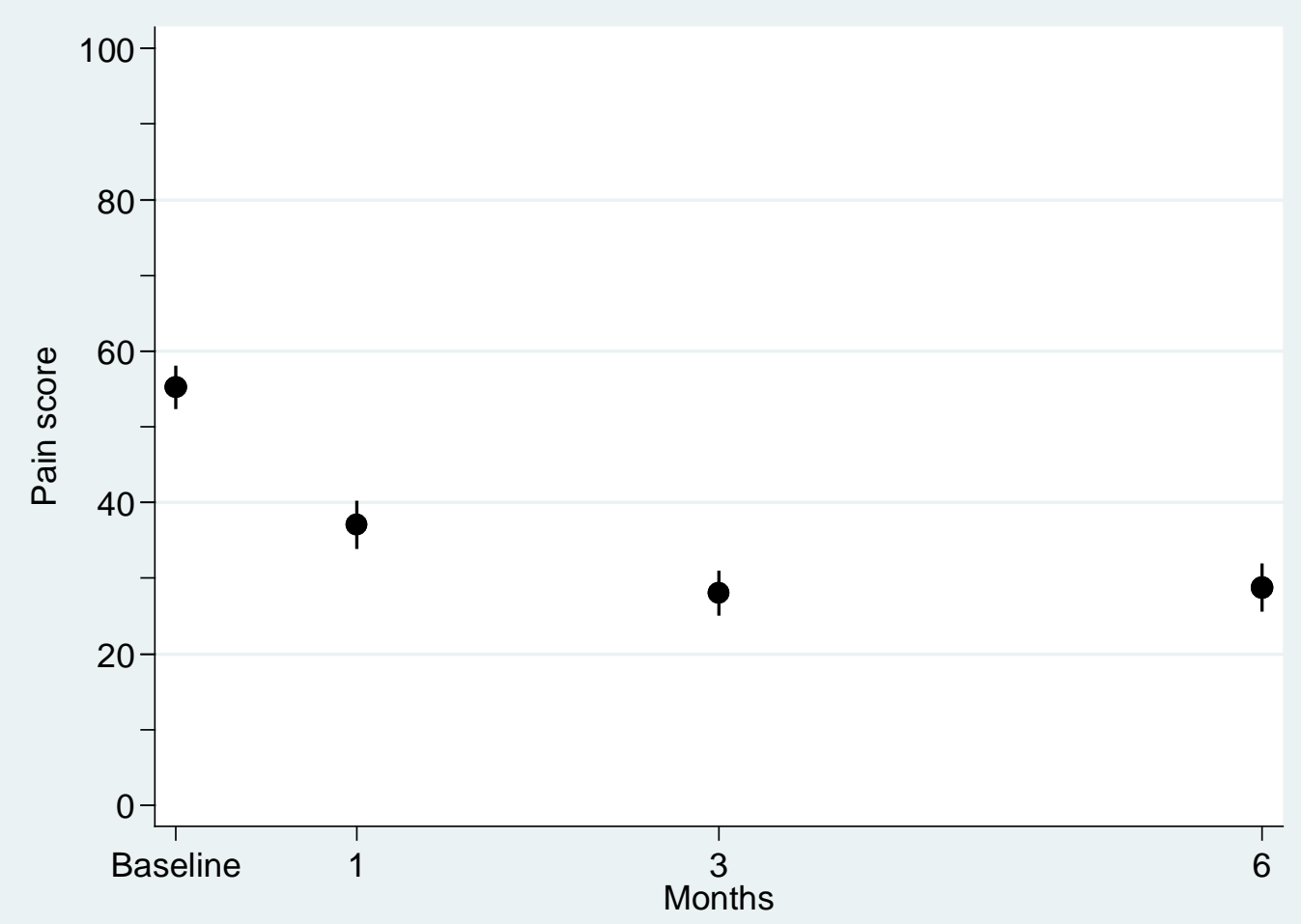

Figure 1. Mean \pm SE of Pain scores over time.

Corresponding reductions in the two disability summary measures were noted over the six months. The mean RMDQ scores reduced significantly from 12.2 (95\% CI: 11.0, 13.3) at inception to a plateau of $7.6(95 \%$ CI: 6.2, 9.0) at three months $(p<0.001)$ and 7.7 (95\% CI: 6.2, 9.2) at six months $(\mathrm{p}<0.001)$ (Fig. 2). The mean Disability Rating Index scores reduced significantly from 46.8 (95\% CI: 42.2, 51.3) at inception to a plateau of 32.7 (95\% CI: 27.6, 37.9) at three months ( $\mathrm{p}<0.001)$ and 30.7 (95\% CI: 25.5, 35.8) at six months $(\mathrm{p}<0.001)$ (Fig. 3). 


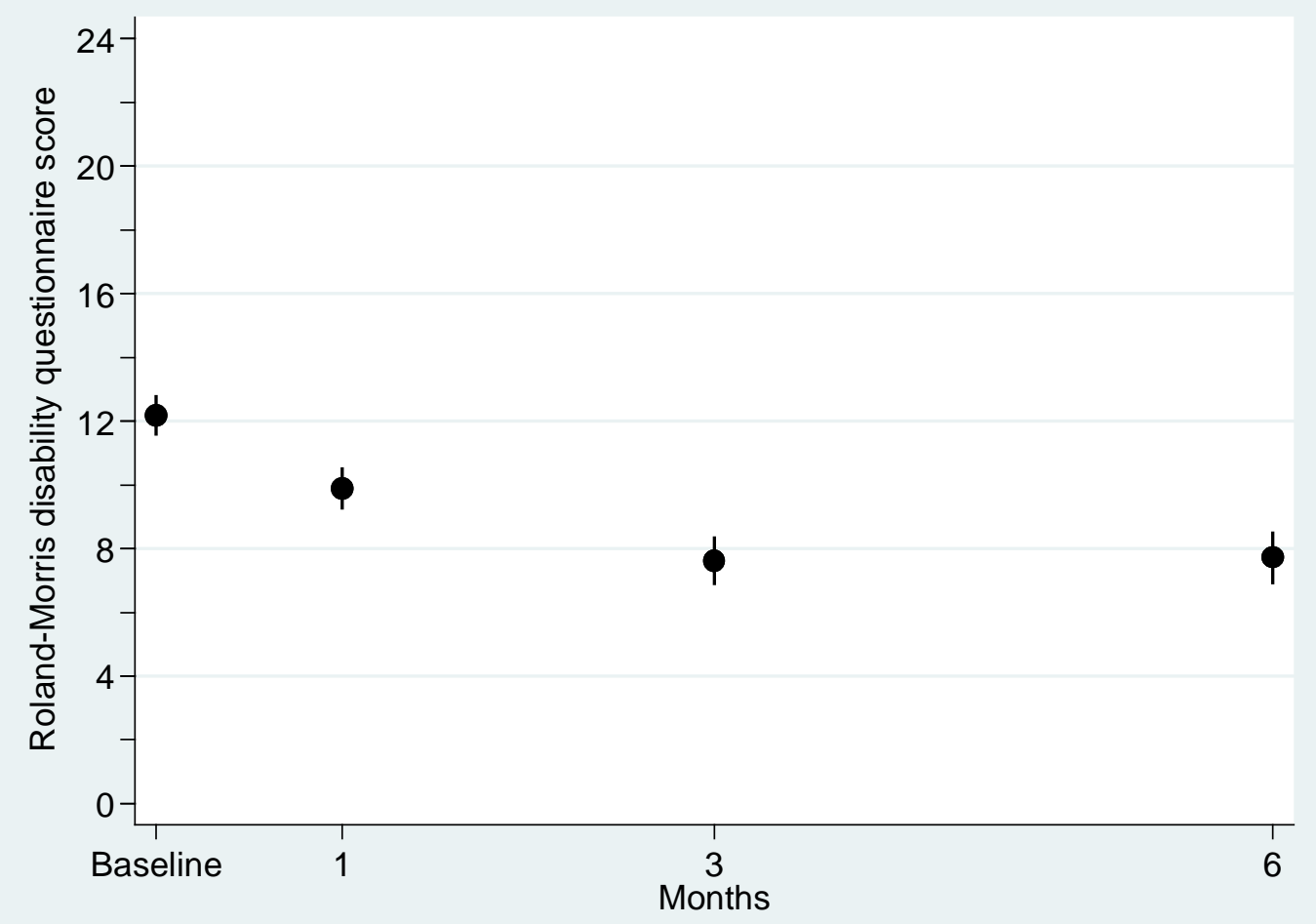

Figure 2. Mean \pm SE of Roland-Morris Disability Questionnaire scores over time. 


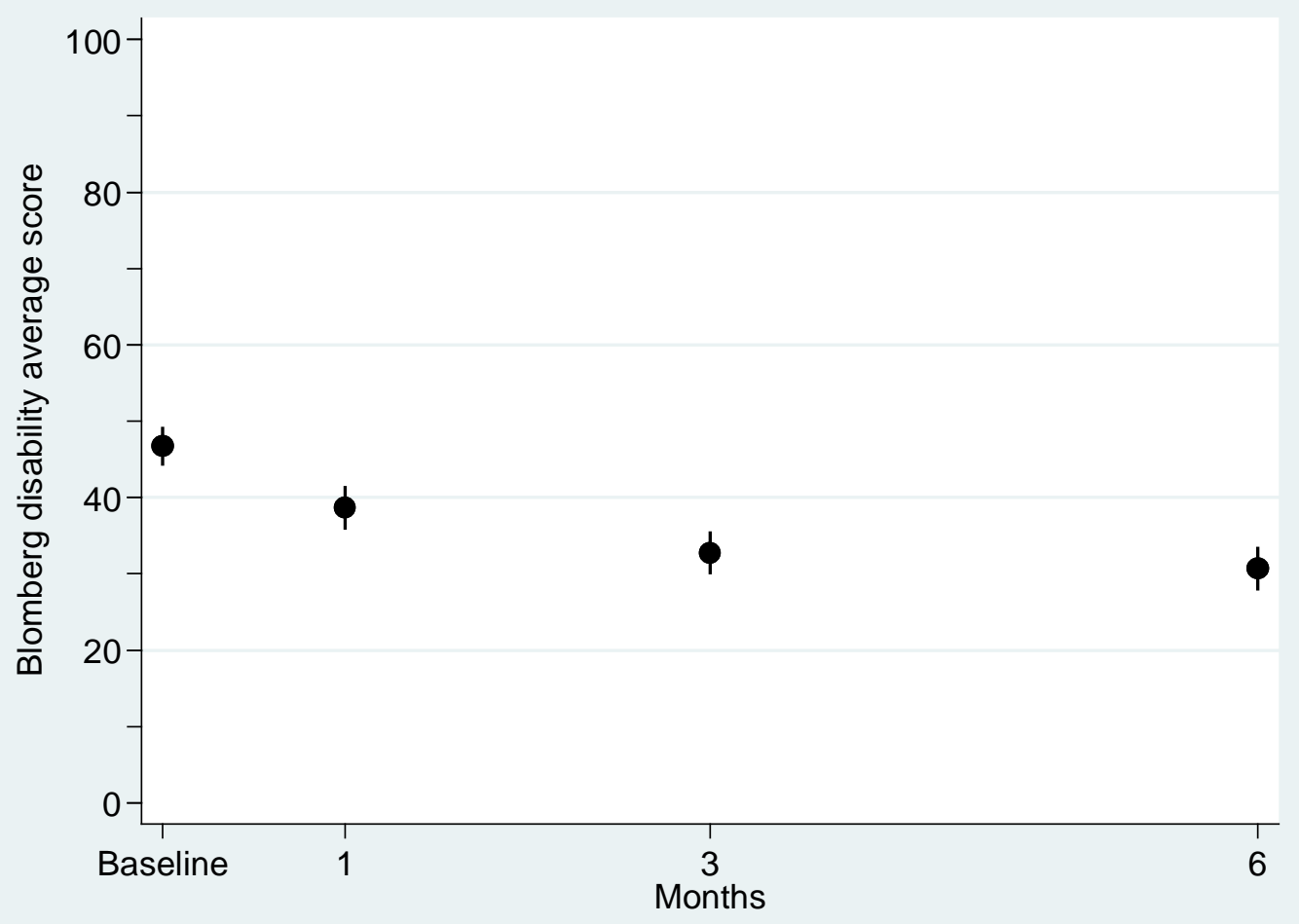

Figure 3. Mean \pm SE of Blomberg disability average scores over time.

Regarding the SF-12 measure, the mean physical component scores increased significantly from 34.4 (95\% CI: 32.6, 36.2) at inception to a plateau of 39.7 (95\% CI: 37.2, 42.1) at three months $(\mathrm{p}<0.001)$ and $39.8(95 \%$ CI: 37.4, 42.1) at six months $(\mathrm{p}<0.001)$ (Fig. 4). The mean mental component scores increased significantly from 45.7 (95\% CI: 43.0, 48.5) at inception to a peak of 51.4 (95\% CI: 48.7, 54.1) at three months $(\mathrm{p}<0.001)$ before significantly decreasing to 47.5 (95\% CI:44.6, 50.5) at six months ( $p=0.02$, compared to three months) to a level that was no longer significantly different to the scores at inception (Fig. 4). 


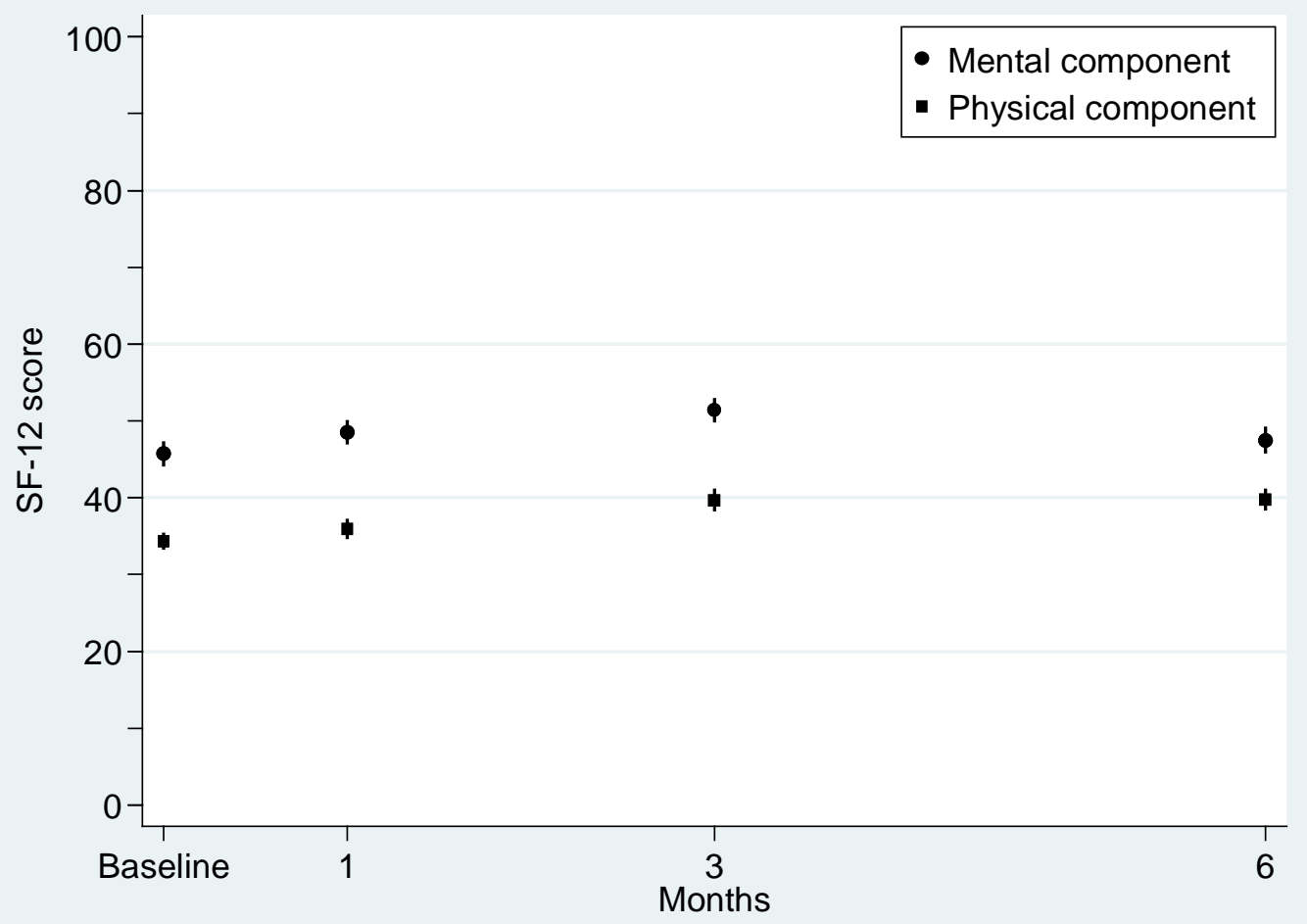

Figure 4. Mean \pm SE of SF-12 mental and physical component scores over time

Table 3. Global assessment of response to treatment and satisfaction with treatment at six months from inception.

\begin{tabular}{ll}
\hline Patient Response & $\mathbf{n}(\%)$ \\
Global assessment & $11(20 \%)$ \\
\hline Very Much Improved & $17(31 \%)$ \\
Much Improved & $16(29 \%)$ \\
Minimally Improved & $8(15 \%)$ \\
No Change & $3(5 \%)$ \\
Minimally Worse & $0(0 \%)$ \\
\hline
\end{tabular}




\begin{tabular}{ll}
\hline \multicolumn{1}{l}{ Very Much Worse } & $0(0 \%)$ \\
Satisfaction & $12(22 \%)$ \\
Extremely satisfied & $13(24 \%)$ \\
Very satisfied & $8(15 \%)$ \\
Somewhat satisfied & $12(22 \%)$ \\
Neutral & $5(9 \%)$ \\
Somewhat dissatisfied & $1(2 \%)$ \\
Very dissatisfied & $3(6 \%)$ \\
Extremely dissatisfied & \\
\hline
\end{tabular}

The results of the global assessment of response to treatment and satisfaction with treatment at six months from inception are presented in table 3. Eighty percent of patients improved and 20\% reported that they were unchanged or minimally worse. There were moderate correlations between the change in pain and the global assessment ( $r=0.53)$ and between change in disability and global assessment $(r=0.53)$.

Sixty-one percent were satisfied with treatment, $17 \%$ were dissatisfied and $22 \%$ were neutral. There was a moderate correlation $(\mathrm{r}=0.43)$ between the change in pain and satisfaction and between change in disability and satisfaction $(\mathrm{r}=0.46)$. There was a good correlation between the global assessment and satisfaction ( $\mathrm{r}=0.74)$.

Subgroup analysis and predictors of response 
A post-hoc analysis of subgroups within the cohort showed some differences in pain and disability at inception. Those with back pain and leg pain had higher RMDQ levels than those with back pain alone ( $p=0.01)$. Similarly, smokers had higher pain $(p=0.046)$ and RMDQ ( $\mathrm{p}=0.01)$ levels than non-smokers. Patients treated by either doctor GH or doctor MY had higher pain scores than those treated by doctor SM $(\mathrm{p}=0.002)$. However there were no differential responses over time in any of these subgroup analyses.

Those with one or two previous episodes of back pain showed greater reductions in pain over six months than those with no previous episodes or those with more than two previous episodes $(\mathrm{p}=0.034)$. Those currently off work had higher disability levels than those currently at work at inception $(\mathrm{p}=0.001)$ and also showed greater reductions in both pain $(\mathrm{p}<0.001)$ and disability $(\mathrm{p}=0.045)$ over six months. Those with pain up to three months showed greater reductions in both pain $(\mathrm{p}=0.003)$ and disability $(\mathrm{p}<0.001)$ over six months than those with pain longer than three months.

\section{DISCUSSION}

This is the first trial to examine multimodal management of LBP in Australia that has included chronic persistent pain patients. The cohort examined were predominantly middle-aged people with very chronic pain and a history of recurrent episodes of back pain. Most participants were currently working, had back and leg pain, had morning stiffness and were non-smokers. There were also more females in this cohort. The primary outcome measures (pain intensity and the RMDQ) showed statistically and 
clinically significant reductions in pain and disability over three months with maintenance in these reductions at six months. Similar significant reductions were also seen in the Disability Rating Index over the study period. The best reductions in pain and disability were seen in participants who were currently not working, had pain less than three months and had one or two previous episodes of back pain.

There may have been some selection bias in this trial as it was a non-consecutive case series due to time constraints and patient refusals. We were unable to collect data on eligible non-participants. Also follow-up rates at three and six months were slightly under the acceptable threshold of $80 \%$. Whilst the treating doctor was not a predictor of response in this trial, these results may be difficult to generalise. The three treating doctors in this study had post-graduate training in musculoskeletal medicine, the NMMI and the STAYAC algorithm. There was however, some variation in the treatment modalities utilised by each doctor (e.g. one doctor did not refer any participants to a physiotherapist).

The Ausback study compares favourably with other studies on chronic LBP, however comparisons are tempered by the differing cohort characteristics including that $19 \%$ of the Ausback study cohort had acute pain at inception. A randomised control trial set in the UK investigating the efficiency of musculoskeletal physiotherapy on chronic low back disorder[20] followed 346 patients randomised into a 10 week spinal stabilisation program, manual therapy group or minimal intervention group. Best results were 
achieved with the stabilisation program with pain reducing from a mean of 45.7 at inception to 28.8 and 23.2 at three and six months respectively.

Another randomised control trial in the UK[21] looked at delivering a 16 hour group program of education and exercise using a cognitive behavioural therapy approach. This showed modest reductions in pain levels on a $100 \mathrm{~mm}$ VAS from a mean of 44.9 at inception to 29.1 and 26.1 at three and nine months respectively and in RMDQ scores from 10.6 at baseline to 7.4 and 6.5 .

Acupuncture has been used widely for LBP. A recent trial in the USA with patients with between three and 12 months of LBP[22], those treated with individualized acupuncture showed a reduction in mean RMDQ scores reduced from 10.8 to 6.4 and 6.8 at two and six months respectively. The reduction in these scores, with 10 treatment visits, was very similar to those observed in our cohort, with only three visits.

Multidisciplinary therapy based on intensive exercises has been utilised for chronic LBP with intensive rehabilitation combined with functional restoration giving better results than less intensive interventions.[23] However the absolute and percentage pain reductions at six months reported in recent trials of intensive multidisciplinary programs for chronic LBP have been lower than in our study.[24, 25] Comparison of reductions in disability are limited by the use of different outcome measures. 
In contrast, the Swedish study[8] examining the STAYAC algorithm for treatment of acute and sub-acute LBP showed greater reductions in pain and disability than our cohort. Mean pain scores (on a 100-point VAS) reduced from 54.7 at baseline to 16.2 after 10 weeks of treatment. Over the same period the disability rating index reduced from 57.8 to 20.1. Attributes of the Swedish study that could explain the better response include the shorter duration of back pain, better adherence to the STAYAC algorithm, the more frequent use of standardized manipulation and injection techniques and differing sociocultural characteristics between Sweden and Australia.

Chronic LBP is more costly to the community and has the added drawback in that it would seem to be more resistant to treatment than acute LBP. This study does show however that a 'blended' pragmatic and evidence-based approach which involves multiple treatment interventions is associated with significant and clinically important improvements in both pain and disability scores. The contribution of the natural history of back pain to the observed improvements is difficult to estimate, but what is known is that natural history contributes more when the pain is of a shorter duration (Smedley, 1998). However the chronic nature of back pain in over $80 \%$ of this series may have diminished this effect.

Which of the multiple interventions used in this study are the "key" ones remains uncertain.. It may be that the combination of a number of interventions is better than single interventions . Further studies with control of comparison groups are needed to resolve these questions. 
Future studies of this approach should also collect data on costs to allow an analysis of cost-efficiency and cost-utility. We found that similar clinical improvements to more intensive individual and group programs can occur with relatively small numbers of visits, suggesting that this 'blended' approach could be a more efficient method of treating low back pain. 


\section{REFERENCES}

1. Australian Institute of Health and Welfare. General practice activity in Australia 2006-07.

2. $\quad$ Buchbinder R, Jolley D, Wyatt M. Breaking the back of back pain MJA 2001; 175:456-457.

3. Briggs AM, Buchbinder R. Back Pain: A National Health Priority Area in Australia? MJA 2009;190(9):499-502.

4. Henschke N, Maher CG, Refshauge KM, et al. Prognosis in patients with recent onset low back pain in Australian primary care: inception cohort study. BMJ 2008; 337:a171.

5. McGurk B, King W, Govind J, Lowry J, Bogduk N. The safety, efficacy and costeffectiveness of evidence-based guidelines for the management of acute low back pain in primary care. Spine 2001;26:2615-22.

6. Indahl A, Velund L, Reikeraas O. Good prognosis for low back pain when left untampered: A randomized clinical trial. Spine 1995;20:473-7. 
7. Karjalainen KA, Malmivaara A, van Tulder MW, Roine R, Jauhiainen M, Hurri H, Koes BW. Multidisciplinary biopsychosocial rehabilitation for subacute low-back pain among working age adults. Cochrane Database of Systematic Reviews 2003, Issue 2. Art. No.: CD002193. DOI: 10.1002/14651858.CD002193.

8. Grunnesjo MI, Bogefeldt JP, Svardsudd KF, Blomberg SI. A randomized controlled clinical trial of stay-active care versus manual therapy in addition to stayactive care: functional variables and pain. J Manipulative Physiol Ther 2004;27(7):43141.

9. Bogduk N, McGuirk B. Medical Management of Acute and Chronic Low Back Pain. An Evidence-Based Approach. Sydney: Elsevier 2002.

10. Raspe H. Management of chronic low back pain in 2007-2008. Curr Opin Rheumatol 2008;20(3):276-81.

11. Blomberg S. A pragmatic strategy for low back pain - an integrated multimodal programme based on antidysfunctional medicine. Musculoskeletal Medicine 2005. 4.4 Oxford press.

12. Price DD, Bush FM, Long S, Harkins SW. A comparison of pain measurement characteristics of mechanical visual analogue and simple numerical rating scales. Pain. 1994;56(2):217-26. 
13. Farrar JT, Young JP Jr., LaMoreaux L, Werth JL, Poole RM. Clinical importance of changes in chronic pain intensity measured on an 11-point numerical pain rating scale. Pain. 2001;94(2):149-58.

14. Riddle DL, Stratford PW, Binkley JM. Sensitivity to Change of the RolandMorris Back Pain Questionnaire: Part 2. Physical Therapy 1998;78(11):1197-207.

15. Salén B, Spangfort E, Nygren Ă, Nordemar R. The disability rating index: an instrument for the assessment of disability in clinical settings. J Clin Epidemiol $1994 ; 47: 1423-34$

16. Ware J, Jr., Kosinski M, Keller SD. A 12-Item Short-Form Health Survey: construction of scales and preliminary tests of reliability and validity. Med Care 1996;34(3):220-33.

17. Gordon S, Ameen V, Bagby B, Shahan B, Jhingran P, Carter E. Validation of irritable bowel syndrome Global Improvement Scale: an integrated symptom end point for assessing treatment efficacy. Dig Dis Sci 2003;48(7):1317-23.

18. Goldsmith CH, Boers M, Bombardier C, Tugwell P. Criteria for clinically important changes in outcomes: Development, scoring and evaluation of rheumatoid 
arthritis patient and trial profiles. OMERACT Committee. J Rheumatol 1993;20(3):561-

5.

19. Juniper EF, Guyatt GH, Willan A, Griffith LE. Determining a minimal important change in a disease-specific Quality of Life Questionnaire. J Clin Epidemiol 1994;47(1):81-7.

20. Goldby LJ, Moore AP, Doust J, Trew ME. A randomized controlled trial investigating the efficiency of musculoskeletal physiotherapy on chronic low back disorder. Spine 2006;31(10):1083-93.

21. Johnson RE, Jones GT, Wiles NJ, Chaddock C, Potter RG, Roberts C, et al. Active exercise, education and cognitive behavioral therapy for persistent disabling low back pain: A randomized controlled trial. Spine 2007;32(15):1578-85.

22. Cherkin DC, Sherman KJ, Avins AL, Erro JH, Ichikawa L, Barlow WE, et al. A randomized trial comparing acupuncture, simulated acupuncture, and usual care for chronic low back pain. Arch Intern Med 2009;169(9):858-66.

23. Bogduk N, McGuirk B. Medical management of acute and chronic low back pain: an evidence-based approach. Amsterdam: Elsevier; 2002. 
24. Lang E, Liebig K, Kastner S, Neundorfer B, Heuschmann P. Multidisciplinary rehabilitation versus usual care for chronic low back pain in the community: Effects on quality of life. Spine 2003;3(4):270-6.

25. Kaapa EH, Frantsi K, Sarna S, Malmivaara A. Multidisciplinary group rehabilitation versus individual physiotherapy for chronic nonspecific low back pain: a randomized trial. Spine 2006;31(4):371-6.

26. Smedley J, Inskip H, Cooper C, Coggon D. Natural history of low back pain. A longitudinal study in nurses. Spine. 1998;23(22):2422-6. 